\title{
Effect of The Isotiazole Adjuvants in Combination With Cisplatin in Chemotherapy of Neuroepithelial Tumors: Experimental Results and Modeling
}

Vladimir Potkin ( $\nabla$ potkin@ifoch.bas-net.by)

Institute of Physical and Organic Chemistry

Aliaxandr Pushkarchuk

Institute of Physical and Organic Chemistry

Alexandra Zamaro

Institute of Physiology National Academy of Sciences of Belarus

Hongwei Zhou

Jiaxing University

Sergey Kilin

B.I. Stepanov Institute of Physics

\section{Sergey Petkevich}

Institute of Physical and Organic Chemistry

Iryna Kolesnik

Institute of Physical and Organic Chemistry

Dominik Michels

King Abdullah University of Science and Technology

Dmitry Lyakhov

King Abdullah University of Science and Technology

Vladimir A. Kulchitsky

Institute of Physiology National Academy of Sciences of Belarus

\section{Research Article}

Keywords:

Posted Date: March 7th, 2022

DOI: https://doi.org/10.21203/rs.3.rs-1358521/v1

License: (c) (1) This work is licensed under a Creative Commons Attribution 4.0 International License.

Read Full License 
Page $2 / 21$ 


\section{Abstract}

Chemotherapy is one of the main treatment options for cancer, but it is usually accompanied with negative side effects. The classical drugs combination with synergistic adjuvants can be the solution to this problem, allowing reducing therapeutic dose. Elucidating the mechanism of adjuvant action is of key importance for selection of the optimal agent. Here we examine the system drug-adjuvant to explain observed effect in practice. We used the first line drug - cisplatin. Morpholinium and 4methylpiperazinium 4,5-dichloro isothiazol-3-carboxylates were selected as adjuvants. The study of cisplatin - adjuvant system was carried out by quantum chemical modeling with DFT method. It turned out that adjuvants form conjugates with cisplatin that leads to relocation of frontier molecular orbitals as well as increase of conjugate's dipole moment. It results in change of the character of interaction with DNA and increase of bioactivity of system. The data obtained are the basis for expanding the studies to include other drugs and adjuvants. Oncologists will have opportunity to use "classical" chemotherapy drugs in combination with synergists for those patients who have not been previously recommended to such treatment because of pronounced toxic side effects.

\section{Introduction}

The problem of radical treatment of malignant neoplasms has not yet been resolved [1]. Three main methods dominate in clinical oncology: surgical removal of tumor, radiation therapy and chemotherapy [2]. All these methods are often used in different combinations to improve treatment effectiveness. Our research focuses on one of these approaches - chemotherapy, which still remains the main method of cancer treatment. The choice of this particular section of clinical oncology is based on significant progress in creation of anticancer chemotherapy drugs, especially in those nosological forms of tumors when it is impossible to use surgical or radiation methods [3]. Successful use of chemotherapy is hindered by a number of factors; coping with them will increase effectiveness of anticancer therapy and reduce mortality in oncology. Nonselective toxicity of used substances causing fatal damage to both tumor and all other rapidly proliferating cells in patient's body is one of the main disadvantages of chemotherapy [4]. In this regard, improvement of tumor chemotherapy includes both the search for new effective antineoplastic targeted agents and development of ways to reduce side effects, primarily toxicity of anticancer therapy. International protocols recommend limiting single and total dose as well as the number of courses to weaken side effects of chemotherapy drugs, but compliance with these conditions is accompanied with natural weakening of anticarcinogenic effects and decrease in treatment effectiveness. The use of synergistic adjuvants in combined chemotherapy of tumors in order to enhance antitumor effects and reduce therapeutic dosages of official chemotherapy drugs is one of the solutions to this problem. Such tactics is aimed at keeping recognized chemotherapy drugs in the list as their development is extremely expensive, and also at protecting patient's organism weakened by tumor process from side effects of cytostatics.

The team of authors of the article has been conducting research on molecular design of structures, synthesis and biotesting of new synergistic adjuvants in compositions with first-line anticancer drugs for 
a number of years. As a result, it was found that isothiazole derivatives are promising candidates for synergist's role. Isothiazole (1,2-thiazole) nucleus has high potential of biological activity and is structural part of a wide range of synthetic bioactive substances [5].

We found that many isothiazole derivatives have potentiating effect in binary mixtures with bioactive compounds, including antitumor agents [6-8]. These are represented by 4,5-dichloroisothiazole-3carboxylic acid, its salts, amides, esters, carbamates, urea, metal complexes with 4,5-dichloroisothiazole moieties, etc. Observed potentiating effect is so pronounced in some cases that it makes possible to reduce the dose of chemotherapy drug by tens of times in in vitro experiments on $\mathrm{C} 6$ glioma culture cells or on primary culture cells from patient neuroepithelial tumor. Thus, an experimental substantiation of methodology is being formed for creating a new generation of anticancer drugs based on combination of existing chemotherapy substances with adjuvants recommended in the process of studies [9].

It is advisable to identify key factors that determine synergistic effect of an adjuvant in a binary mixture, i.e., the mechanism of its manifestation at the molecular level for the purposeful development of new synergists and their compositions with anticancer drugs. This was the main goal of our research.

The co-authors of the article previously hypothesized that inhibitory effect on cyclin-dependent kinases (Cdk2) or VEGFR2 tyrosine kinase is one of the mechanisms of synergism of isothiazole derivatives [9]. The ability to integrate into Cdk2 sites was established by modeling using the docking procedure [6]. The authors are aware that the stated explanation is only one of hypotheses that require verification and proof. In continuation of studies on the problem of synergism, this work represents a quantum-chemical analysis and modeling of "chemotherapy drug - isothiazole adjuvant" system. Cisplatin - (SP-4-2)diamminedichloroplatinum (CPt), widely used in tumor therapy, was chosen as a chemotherapy drug [10]. We took available water-soluble morpholine salt of 4,5-dichloroisothiazole-3-carboxylic acid - morpholin4-ium 4,5-dichloroisothiazole-3-carboxylate (MS) as an adjuvant, which exhibits strong synergistic effect in composition with cisplatin, as previously established by in vitro testing on cultures of neuroepithelial tumor cells [6]. Specially synthesized analogue was taken for comparison: 4-methylpiperazin-1-ium 4,5dichloroisothiazole-3-carboxylate (PS), which differs from MS only by the fact that morpholine fragment of the salt is replaced by an N-methylpiperazine residue (Fig. 1), which is also a pharmacophore fragment [11].

\section{Experimental Section}

\subsection{Materials and methods}

IR spectra were recorded on the Fourier spectrophotometer Thermo Nicolet Protege 460 with $\mathrm{KBr}$ pellets. NMR ${ }^{1} \mathrm{H}$ and ${ }^{13} \mathrm{C}$ spectra were registered on the Bruker Avance 500 spectrometer (500 and $125 \mathrm{MHz}$ respectively) in $\mathrm{CDCl}_{3}$. Internal standard - residual signals of solvent $\mathrm{CDCl}_{3}: 7.26 \mathrm{ppm}$ for ${ }^{1} \mathrm{H}, 77.2 \mathrm{ppm}$ for ${ }^{13} \mathrm{C}$. Assignment of signals in the ${ }^{13} \mathrm{C}$ NMR spectra was performed using the DEPT procedure. Elemental analysis was performed on the automatic Vario MICRO cube CHNS-analyzer. Melting points were 
determined on the Kofler apparatus. Monitoring of reaction progress and assessment of purity of synthesized compounds were done by TLC on Merck Millipore Silica gel 60 F254 plates, eluent $\mathrm{Et}_{2} \mathrm{O}$, visualization by iodine stain.

Cisplatin was purchased from West-Ward Pharmaceuticals Corp., USA. Reagents and solvents were supplied by Aldrich Chemical Co. (China) and used without additional purification. 4,5Dichloroisothiazole-3-carboxylic acid was prepared by previously developed by us method [12].

PS synthesis was carried out by 4,5-dichloroisothiazole-3-carboxylic acid reaction with $\mathrm{N}$ methylpiperazine (Fig. 2), following the procedure used to prepare MS derivative [6].

\subsection{Procedure for synthesis of PS and its spectral data}

To a solution of $0.99 \mathrm{~g} \mathrm{(5} \mathrm{mmol)} \mathrm{of} \mathrm{4,5-dichloroisothiazole-3-carboxylic} \mathrm{acid} \mathrm{in} 25 \mathrm{ml}$ of dry methanol, $0.50 \mathrm{~g}(5 \mathrm{mmol})$ of $\mathrm{N}$-methylpiperazine was added at $20^{\circ} \mathrm{C}$, and the mixture was stirred at this temperature for $2 \mathrm{~h}$. The solvent was distilled off in a vacuum to dryness, the residue is dissolved in $5 \mathrm{ml}$ of dichloromethane. Then hexane was added in portions $(7 \times 5 \mathrm{ml})$ with stirring - after that an oil dropped out. The solvent was distilled off slowly under reduced pressure to $5 \mathrm{ml}$ and the oil passed into a solid precipitate, which was filtered off, washed with hexane and dried in a vacuum. Yield $1.44 \mathrm{~g}(97 \%)$, white solid, m.p. $119-120^{\circ} \mathrm{C}$.

The structure of obtained 4-methylpiperazine 4,5-dichloroisothiazole-3-carboxylate (PS) was confirmed by the data of $\mathrm{IR},{ }^{1} \mathrm{H}$ and ${ }^{13} \mathrm{C}$ NMR spectra and elemental analysis.

IR spectrum, v, cm-1: 2991, 2974, 2948, 2851, 2803, 2770, 2718, 2632, 2468, 1635, 1574, 1454, 1425, $1394,1342,1312,1283,1192,1145,1098,1082,1048,1003,966,846,825,799,732,600,444$.

NMR ${ }^{1} \mathrm{H}$ spectrum $\left(500 \mathrm{MHz}, \mathrm{CDCl}_{3}\right), \delta, \mathrm{ppm}: 2.26 \mathrm{~s}\left(3 \mathrm{H}, \mathrm{CH}_{3}\right), 2.62 \mathrm{t}\left(4 \mathrm{H}, 2 \mathrm{CH}_{2} \mathrm{NCH}_{3}, J=4.7 \mathrm{~Hz}\right), 3.18 \mathrm{t}$ $\left(4 \mathrm{H}, 2 \mathrm{CH}_{2} \mathrm{NH}_{2}^{+}, J=4.9 \mathrm{~Hz}\right), 9.86$ b.s. $\left(2 \mathrm{H}, \mathrm{NH}_{2}^{+}\right)$.

NMR ${ }^{13} \mathrm{C}$ spectrum $\left(125 \mathrm{MHz}, \mathrm{CDCl}_{3}\right)$, ठ, м.д.: $43.33\left(2 \mathrm{CH}_{2} \mathrm{NH}_{2}{ }^{+}\right), 45.90\left(\mathrm{CH}_{3}\right), 51.71\left(2 \mathrm{CH}_{2} \mathrm{NCH}_{3}\right), 124.03$, $148.48,162.49,165.18\left(4 C_{\text {quat }}\right)$.

Elemental analysis. Calculated for $\mathrm{C}_{9} \mathrm{H}_{13} \mathrm{Cl}_{2} \mathrm{~N}_{3} \mathrm{O}_{2} \mathrm{~S}$, \%: C 36.25, H 4.39, N 14.09, S 10.75. Found, \%: C 36.19, H 4.50, N 14.11, S 10.68 .

\subsection{MTT testing}

C6 rat glioma cells have been acquired from the Institute of Cytology of the Russian Academy of Sciences, Saint Petersburg (http://www.cytspb.rssi.ru, http://www.incras.ru). Determination of proliferative activity of rat $\mathrm{C} 6$ glioma cells after addition of cisplatin, new PS compound, and their binary mixture was carried out using MTT test by assessing optical density of medium, which is directly proportional to the number of living cells. The 3-(4,5-dimethylthiazol-2-yl)-2,5-diphenyl-2H-tetrazolium 
bromide (MTT) assay is widely used for determination of cell viability and proliferation. MTT, a yellow tetrazole, is reduced to purple formazan in living cells. We strictly adhered to the Merck MTT protocol (link attached) https://www.sigmaaldrich.com/RO/en/technical-documents/protocol/cell-culture-and-cellculture-analysis/cell-counting-and-health-analysis/cell-proliferation-kit-i-mtt

Studies were carried out in a Labconco laminar (BioHazard, USA) on C6 glioma cells in 96-well plates, the Vybrant MTT Cell Proliferation Assay Kit (Thermo Fisher Scientific, Lithuania) was used, and four series of experiments were performed. The absorbance of the contents of the wells was measured on an automatic biochemical immuno-fermental analyzer ChemWell ${ }^{\circledR} 2910$ (Combi) using ChemWell $\circledast$ software version 6.3 (Revision A), USA. The initial concentration of C6 glioma cells was 5000 cells per well of the plate. The cells were cultured in F10 nutrient medium. We added $10 \%$ bovine fetal serum to the culture medium.

Cisplatin was added at the dose of $0.3 \mathrm{~g} / \mathrm{m}^{2}$, PS was added at the dose of $50 \mu \mathrm{L} / \mathrm{ml}$, as well as in 10 and 100-fold dilutions (0.1 CPt, 0.1 PS and 0.01 Cpt, 0.01 PS, respectively). The effect of both individual compounds and their combinations in different ratios was determined.

\subsection{Quantum chemical methods and modeling procedures}

The Gaussian 16 package software was used to estimation of CPt-MS and CPt-PS conjugates [13]. Preliminary full geometry optimizations of initial structures were performed with the aid of molecular mechanics (MM) by using the same software. All calculations presented in this work were carried out by means of Density Functional Theory (DFT) implemented in Gaussian 16 package too [13]. Geometry optimizations of all molecules were fully optimized at the hybrid exchange-correlation functional with the Coulomb-attenuating method (CAM-B3LYP) used in our DFT calculations. CAM-B3LYP functional combines hybrid qualities of B3LYP $[14,15]$ and the long-range correction presented by Tawada et al [16]. This functional works well for covalent and noncovalent, weak dispersion and hydrogen bonding [17]. Dunning's correlation consistent polarized valence double zeta cc-pVDZ basis set was used $[18,19]$. This basis set has had redundant functions removed and has been rotated in order to increase computational efficiency [20]. The LANL2DZ (Los Alamos National Laboratory 2 double zeta) basis set with effective core potential (ECP) was used for Pt [21, 22].

Polarizable continuum model (PCM, solvent is considered as continuous dielectric medium) was used for solvent phase calculations [23]. The PCM model implements self-consistent reaction field (SCRF) approach and defines solvent polarization in terms of electrostatic potential. Following discussions are based on this method if not noted otherwise. No symmetric constraints were imposed during geometrical optimizations. The energy minima are identified by subsequent frequency calculations. For visualization of quantum chemistry computations Gauss View 6.0.16 and ChemCraft software were used [13, 24].

Calculations of MEP distribution diagrams were performed using DFT / CAM-B3LYP / cc-pvdz / LANL2DZ theory level for optimized CPt-MS and CPt-PS structures.

\section{Results And Discussion}


Obtained results of MTT testing of PS substance, its conjugate CPt-PS and description of the experiment are given in Table 1 in the form of numerical values and are presented in the form of diagrams (Fig. 3). As can be seen, death of tumor cells is about $83 \%$ at the dose of cisplatin $0.3 \mathrm{~g} / \mathrm{m}^{2} .10$-fold reduction in dose leads to decrease up to $33 \%$, and at 100 -fold dilution cisplatin loses its antitumor effect. Individual new isothiazole derivative PS itself in all tested doses did not show any noticeable bioactivity under experimental conditions, but increased cytotoxic effect of cisplatin, although observed effect was lower than for the morpholine MS salt the test results of which were published by us earlier [6]. Thus, addition of $5 \mu \mathrm{g} / \mathrm{ml}$ of adjuvant $(0.01 \mathrm{PS})$ to a 100 -fold dilution of cisplatin $(0.01 \mathrm{CPt})$, when it does not exhibit a cytotoxic effect itself, leads to noticeable antiproliferative activity and death of $\mathrm{C} 6$ glioma cells - up to $18 \%$. We found that the effect of adjuvant addition to cisplatin taken in 10 -fold dilution $(0.1 \mathrm{CPt})$ increases with decreasing dose (quite surprising!), and the cell death for combination $0.1 \mathrm{CPt}+0.1 \mathrm{PS}$ is $38 \%$, and for $0.1 \mathrm{CPt}+0.01 \mathrm{PS}$ it increases up to $48 \%$, which is almost one and a half times higher than for individual cisplatin at such dosage $(32 \%)$.

Table 1

Optical density of $\mathrm{C} 6$ cell culture under the action of cisplatin (CPt), an adjuvant (PS) and their binary mixture at different concentrations and after subtracting the background value (optical density of DMSO)

\begin{tabular}{|c|c|c|c|c|c|c|c|c|c|c|c|}
\hline & C6 & $\begin{array}{l}\text { C6 } \\
+ \\
\text { CPt }\end{array}$ & $\begin{array}{l}\text { C6 } \\
+ \\
0.1 \\
\text { CPt }\end{array}$ & $\begin{array}{l}\text { C6 } \\
+ \\
0.01 \\
\text { CPt }\end{array}$ & $\begin{array}{l}\text { C6 } \\
+ \\
\text { PS }\end{array}$ & $\begin{array}{l}\text { C6 } \\
+ \\
0.1 \\
\text { PS }\end{array}$ & $\begin{array}{l}\text { C6 } \\
+ \\
0.01 \\
\text { PS }\end{array}$ & $\begin{array}{l}\text { C6+ } \\
0.1 \\
\text { CPt }+ \\
0.1 \\
\text { PS }\end{array}$ & $\begin{array}{l}\text { C6+ } \\
0.1 \\
\text { CPt+ } \\
0.01 \\
\text { PS }\end{array}$ & $\begin{array}{l}\text { C6+ } \\
0.01 \\
\text { CPt+ } \\
0.1 \text { PS }\end{array}$ & $\begin{array}{l}\text { C6+ } \\
0.01 \\
\text { CPt+ } \\
0.01 \\
\text { PS }\end{array}$ \\
\hline Mean & 0.34 & 0.06 & 0.23 & 0.35 & 0.33 & 0.33 & 0.33 & 0.21 & 0.18 & 0.28 & 0.31 \\
\hline $\begin{array}{l}\text { Error } \\
\text { of } \\
\text { Mean }\end{array}$ & 0.01 & 0.01 & 0.02 & 0.02 & 0.03 & 0.03 & 0.02 & 0.02 & 0.01 & 0.02 & 0.02 \\
\hline
\end{tabular}

Since the adjuvants (MS and PS) were used in doses when they themselves did not exhibit cytotoxic action, we assumed that synergistic effect appears due to conjugation of cisplatin with isothiazole derivative and subsequent antitumor effect of molecules of resulting conjugate.

In this regard, it would be very informative to determine structural and electronic changes that occur in the system of two conjugated molecules. The information can be obtained as a result of correct quantum chemical calculations. Taking into account chemical and structural features of cisplatin and adjuvants, we assumed that association of their molecules occurs due to non-covalent interactions. To assess structure and strength of cisplatin and adjuvant complexes, as well as their physicochemical characteristics, it is correct to use methods of quantum chemistry, adapted for calculating forces of intermolecular interaction. That, DFT/CAM-B3LYP/cc-pvdz/LanL2DZ(Pt) level of theory was used in our justifications.

We calculated the optimal geometry of molecules, dipole moment, charge distribution, localization and energy characteristics of frontier molecular orbitals (FMO). Calculations were carried out both for individual compounds and their conjugates in two versions: isolated molecules in vacuum and with 
consideration to aqueous medium, which simulates situation in living cells. Molecular structures (Fig. 4) were obtained as a result of calculations with full optimization of all geometric parameters.

Tribak and colleagues have used data of quantum-chemical calculations of electronic structure of compounds to analyze biological activity of these substances $[25,26]$. Biological activity was considered by analogy with chemical activity of molecules. We also tried to apply results of calculations of optimized CPt-MS and CPt-PS structures to interpret the effect of adjuvant and elucidate possible causes of synergism in conjugate "cisplatin - isothiazole derivative". We calculated distribution of frontier molecular orbitals (FMO): Highest Occupied Molecular Orbital (HOMO) and Lowest Unoccupied Molecular Orbital (LUMO) for CPt, CPt-MS and CPt-PS in vacuum and with consideration to aqueous medium. Results of calculating localization of HOMO and LUMO are shown in the form of 3D isosurfaces (Fig. 57). Determination of localization of FMO is important for establishing preferred directions and regions of the molecule for attack by nucleophiles and electrophiles. These are molecular fragments of antitumor agent that interact with target sites as applied to interpretation of biological activity.

First of all, it should be noted that the results of calculations for isolated molecules in vacuum and in an aqueous medium differ significantly. It follows from calculation data that cisplatin molecules (CPt) and isothiazole derivatives (MS and PS) form conjugates with shortened interatomic distances due to noncovalent interactions caused by hydrogen and van der Waals bonds (Fig. 4). Hydrogen bonds are clearly expressed between oxygen atom of isothiazole carboxylate residue and amine fragment of cisplatin $\mathrm{C}=\mathrm{O}_{\mathrm{MS} / \mathrm{PS}} \cdots \mathrm{H}-\mathrm{N}_{\mathrm{CPt}}$ with interatomic distances of $1.79-1.93 \AA$ in vacuum in both conjugates. These connections are absent in aquatic environment. There are some other interplays in structures of conjugates that differ in aqueous medium and vacuum. For example, for both complexes, shortened contacts are observed between chlorine atoms of cisplatin and $\mathrm{CH}$ fragments of morpholine and piperazine $\left(\mathrm{Cl}_{\mathrm{CPt}}{ }^{\cdots} \cdot \mathrm{H}-\mathrm{C}_{\text {morph }}, \mathrm{Cl}_{\mathrm{CPt}} \cdots \mathrm{H}-\mathrm{C}_{\text {pip }}\right)$, which values in aqueous medium $(2.29,3.22,3.43 \AA)$ are less than in vacuum $(3.50,3.87 \AA$ ), and corresponding values for CPt-PS conjugate are higher than for CPt-MS conjugate. However, there is no common single trend in the change in interatomic distances between the corresponding molecular fragments during the transition from vacuum to an aqueous medium. In particular, for CPt-PS conjugate, the $\mathrm{Cl}_{\text {isoth }} \cdots{ }^{\cdots}-\mathrm{N}_{\mathrm{CPt}}$ and $\mathrm{Cl}_{\mathrm{CPt}} \cdots \cdot \mathrm{H}-\mathrm{N}_{\text {pip }}$ values in vacuum are lower than in aqueous medium, in contrast to $\mathrm{Cl}_{\mathrm{CPt}} \cdots \cdot \mathrm{H}-\mathrm{C}_{\text {pip }}$ values. It can only be stated with confidence that mutual arrangement of molecules of heterocycles (MS and PS) and cisplatin (CPt) and their relative orientation in vacuum and in aqueous medium differ significantly. The differences found are of great importance for interpretation and modeling of antitumor effect of substances, since the agent-target interaction in reality takes place in aquatic environment.

Both in vacuum and aqueous medium $\mathrm{HOMO}$ is localized on $\mathrm{Pt}$ and $\mathrm{Cl}$ atoms in individual cisplatin molecule CPt (Fig. 5a,c). LUMO is localized on $\mathrm{NH}_{3}$ groups in vacuum (Fig. 5b) and delocalized throughout the molecule in aqueous medium (Fig. 5d). It is important to know what happens to its molecule when conjugated with adjuvant. It turns out that LUMO is localized on isothiazole heterocycle in both CPt-MS and CPt-PS conjugates in vacuum and aqueous medium. HOMO is localized in CPt-MS 
conjugate, both in vacuum and aqueous medium, on cisplatin molecule with maximum density on Cl-Pt$\mathrm{Cl}$ fragment, same as in individual cisplatin molecule, but the contribution of $\mathrm{Pt}$ atom in aqueous medium in CPt-MS is greater than in individual cisplatin. HOMO is also located on Cl-Pt-Cl fragment in CPt-PS conjugate in vacuum. The picture fundamentally changes in aquatic medium - HOMO is localized on piperazine fragment.

For explanation of results obtained the mechanism of cytotoxic action of cisplatin was taken into account. Despite the differences in interpretation of some precise details, it is believed that cytotoxic effect of cisplatin is due to disruption of DNA functions via binding to purine bases in its molecule. This leads to intra- and inter-stranded DNA cross-linking, which causes DNA damage, impaired replication and transcription, and subsequently apoptosis of cancer cells [27-29].

Localization of HOMO and LUMO determines which fragments of the molecule are preferred for attack by electrophiles and nucleophiles, respectively. The following conclusions can be drawn from the analysis of FMO distribution. Formation of CPt-MS and CPt-PS conjugates leads to displacement of LUMO from cisplatin molecule and its localization on isothiazole heterocycles both in vacuum and aqueous medium. Since LUMO determines interaction with target nucleophilic sites, this type of binding will be realized through isothiazole heterocycle and not through cisplatin molecule. With regard to generally accepted mechanism, we can say that binding to purine bases in CPt-MS and CPt-PS conjugates will proceed with direct participation of adjuvant, which explains its role in manifestation of synergistic effect.

Cross-linking of DNA strands can cause local denaturation and damage to DNA and appearance of new sites, including electrophilic ones. Binding to electrophilic DNA sites determines localization of HOMO in conjugate. Calculations for conjugate molecules in vacuum showed that HOMO in both conjugates is completely localized on cisplatin molecule. Calculations with consideration to aquatic medium revealed differences in localization of HOMO in conjugates. It still remains on cisplatin molecule in CPt-MS, while in CPt-PS it is predominantly localized on PS piperazine residue. This may be one of the reasons for lower activity of CPt-PS compared to CPt-MS.

The energies of frontier molecular orbitals (FMO) are also used as one of characteristics of biological activity of a molecule. The key characteristics of a molecule in FMO theory are difference between the energies of HOMO and LUMO $(\triangle \mathrm{E})$, global hardness and softness of the system ( $\eta$ and $\mathrm{S}$ ) which are treated as descriptors. We performed calculation of descriptors as well as dipole moments for optimized structures CPt, CPt-MS and CPt-PS in vacuum and with consideration to aqueous medium. Obtained values are shown in Table 2. 
Table 2

Calculated DFT method values of descriptors $\triangle \mathrm{E}, \eta$ and $\mathrm{S}$ and dipole moments in vacuum and in aqueous medium for CPt, CPt-MS and CPT-PS

\begin{tabular}{|lllllll|}
\hline & \multicolumn{3}{l}{ In vacuum } & \multicolumn{3}{c|}{ In aqueous medium } \\
\hline Descriptors & CPt & CPt-MS & CPt-PS & CPt & CPt-MS & CPt-PS \\
\hline LUMO $(\mathrm{eV})$ & -0.7946 & -0.7215 & -0.4042 & -0.3053 & -0.5667 & -0.5550 \\
\hline HOMO $(\mathrm{eV})$ & -7.8982 & -8.0759 & -8.0815 & -8.2420 & -8.2638 & -8.0346 \\
\hline$\Delta \mathrm{E}(\mathrm{eV})$ & 7.1035 & 7.3544 & 7.6773 & 7.9367 & 7.6971 & 7.4796 \\
\hline$\eta$ & 3.5518 & 3.6772 & 3.8386 & 3.9683 & 3.8486 & 3.7398 \\
\hline $\mathrm{S}$ & 0.1408 & 0.1360 & 0.1303 & 0.1260 & 0.1299 & 0.1337 \\
\hline Dipole Moment (Debye) & 10.954 & 3.271 & 2.502 & 16.438 & 19.032 & 18.990 \\
\hline
\end{tabular}

The differences in calculated descriptors values for CPt, CPt-MS and CPt-PS are small. However, there is tendency to decrease in $\triangle \mathrm{E}$ when going from $\mathrm{CPt}$ to conjugates in calculations with consideration to aqueous medium. This indicates an increase in reactivity of the system, which in our case means more active binding with DNA. There is a slight decrease in global hardness and a small increase in global softness in CPt - CPt-MS - CPt-PS series. It should be noted that the opposite situation is observed for calculations in vacuum.

The difference in values of dipole moments is very significant, and in vacuum dipole moment of conjugates is greatly reduced in comparison with cisplatin (3.27, 2.50 and $10.95 \mathrm{D}$ respectively), but in aqueous medium, on the contrary, dipole moment of conjugates is higher than that of cisplatin (19.03, 18.99 and 16.44 respectively), which indicates a greater polarity of conjugate molecules. We have noted such differences in the data for isolated molecules in vacuum and aqueous medium during FMO analysis. This represents great influence of environment (water) on structure and properties of the objects under consideration.

We used one more descriptor for the analysis of "cisplatin - isothiazole derivative" system - molecular electrostatic potential (MEP) [30]. It allows evaluating electrostatic component of the energy of intermolecular interactions and is clearly presented in the form of color diagrams. The MEP chart can be used to predict reactivity and active sites for interactions. Negative electrostatic potential corresponds to proton attraction by total electron density in a molecule, that is, protonation of a molecule (shades of red), and positive electrostatic potential corresponds to proton repulsion by atoms (shades of blue). Potential increases in the following order: red < orange < yellow < green < blue. The MEP distribution diagrams in different formats calculated with consideration of aquatic environment are shown in Fig. 8. Calculation results show that regions with negative potential are located on oxygen atoms of carboxyl group $(-0-C$ $=0$ ) of isothiazole derivative. Areas with positive potential are localized around amino groups of cisplatin. Thus, conjugation of adjuvant with cisplatin results in formation of molecular substrate in which types of electrostatic interactions are shared between cisplatin and isothiazole ligand. 


\section{Conclusions}

In general, the following conclusions can be drawn from the obtained results.

The results of quantum-chemical calculations of the optimal geometry and electronic structure of molecules differ for isolated molecules in vacuum and in an aqueous medium and allow revealing one of possible reasons for the effect of adjuvant in combined chemotherapy of tumors, namely:

1. Adjuvants of isothiazole series shape conjugates with cisplatin with non-covalent interactions between molecules due to hydrogen, van der Waals and electrostatic bonds. The resulting conjugate of two molecules acts as a single unit.

2. Formation of conjugates leads to relocation of frontier molecular orbitals between cisplatin molecules and adjuvant. LUMO localizes on isothiazole heterocycle, which appears to be preferred for binding with DNA molecules. This fact allows explaining the effect of adjuvant in used composition.

3. Unlike LUMO, HOMO is localized on cisplatin molecule in CPt-MS conjugate, while in CPt-PS conjugate it is located on cisplatin molecule in vacuum and mainly on piperazine PS fragment in aqueous medium. These features are one of the reasons for lower activity of CPt-PS compared to CPt-MS.

4. Convergence of energies of frontier molecular orbitals, small changes in global hardness indicate increase in reactivity of conjugates due to association of cisplatin with adjuvant, which is accompanied with increase in cytotoxic effect.

5. Increase in dipole moment of conjugates in aqueous medium, i.e., polarity of their molecules, in comparison with individual cisplatin, also promotes activation of cytotoxic agent binding to the target, which enhances manifestation of adjuvant effect.

6. Diagrams of MEP distribution in conjugates demonstrate significant role of isothiazole ligand in distribution of electrostatic potential in the system.

Thus, results of quantum-chemical calculations revealed various aspects of isothiazole adjuvant effect in conjugated state with cisplatin on manifestation of cytotoxic activity against tumor cells. We are guided by the concept of using different adequate approaches and synergistic effects to achieve the positive results in treatment of malignant neoplasms [31]. Implementation of proposed methodology in the process of experimental research in clinical practice is able to turn hypothesis into reality.

The data obtained in experimental studies are the basis for expanding the scope of such studies to include other chemotherapy drugs that are used in modern oncology, as well as developments with the study of other adjuvants' effects. Such tactics will result in expansion of indications for the use of chemotherapy drugs recommended by international protocols in reduced dosages. Oncologists will have opportunity to use "classical" chemotherapy drugs in combination with synergists for those patients who have not previously been recommended to such prescriptions because of pronounced toxic side effects. 
Moreover, there is an obvious economic effect of using "classical" chemotherapy drugs in comparison with expensive procedure for development, testing and introduction of new chemotherapeutics.

\section{Declarations}

\section{Data availability}

Biotesting data are in body text.

An array of data obtained as a result of quantum chemical calculations and modeling is available at the link https://www.bsuir.by/m/12_100229_1_160639.zip.

\section{Acknowledgments}

This work was carried out with partly financial support from the Belarusian State Scientific Research Program "Convergence 2025", National Academy of Sciences of Belarus. All Gaussian 16 computations were performed on KAUST's Ibex HPC, Saudi Arabia. The authors thank the KAUST Supercomputing Core Lab team for assistance with tasks execution on Skylake nodes.

\section{Author contributions}

All authors designed the study, interpreted the results, and contributed to the writing of the manuscript. VP supervised the study in chemical area, VK supervised the study in medical direction, $\mathrm{HZ}$ - in biochemical field and SK - in physical area. AP, DM, and DL developed the model and did the quantum chemical calculation and modeling. AZ carried out MTT testing, SP performed chemical synthesis and identification of adjuvants. VP, and VK summarized and analyzed all obtained data and together with IK drafted the manuscript. Everybody had access to the data and the final responsibility to submit for publication. All authors read and edited the manuscript.

\section{Competing interests}

The authors declare no competing interests.

\section{References}

1. Sung, H., Ferlay, J., Siegel, R. L., Laversanne, M., Soerjomataram, I., Jemal, A. \& Bray, F. Global Cancer Statistics 2020: GLOBOCAN Estimates of Incidence and Mortality Worldwide for 36 Cancers in 185 Countries. CA Cancer J. Clin. 71, 209-249 https://doi.org/10.3322/caac.21660 (2021).

2. Rivoirard, R., Vallard, A., Langrand-Escure, J., Ben Mrad, M., Wang, G., Guy, J.-B., Diao, P., Dubanchet, A., Deutsch, E., Rancoule, C., \& Magne, N. Thirty years of phase I radiochemotherapy trials: Latest development. Eur. J. Cancer 58, 1-7, https://doi.org/10.1016/j.ejca.2016.01.012 (2016).

3. Cortinovis, D., Bidoli, P., Canova, S., Colonese, F., Gemelli, M., Lavitrano, M. L., Banna, G.L., Liu, S.,V. \& Morabito, A. Novel Cytotoxic Chemotherapies in Small Cell Lung Carcinoma. Cancers 13, 1152 
https://doi.org/10.3390/cancers13051152 (2021).

4. Crivelli, F., Perfetti, E., Sartorello, B., Cantele., V., Ostano, P., Ilardi., F., Di Massimo, D. S. \& Clerico, M. Survey on symptoms in patients receiving chemotherapy in an Italian department of oncology: a comparison between period prevalences. Supp. Care Cancer 29, 255-261 https://doi.org/10.1007/s00520-020-05486-x (2021).

5. Kletskov, A. V., Bumagin, N. A., Zubkov, F. I., Grudinin, D. G. \& Potkin, V. I. Isothiazoles in the Design and Synthesis of Biologically Active Substances and Ligands for Metal Complexes. Synthesis 52, 159-188 https://www.thieme-connect.com/products/ejournals/abstract/10.1055/s-0039-1690688 (2020).

6. Kulchitsky, V. A., Potkin, V. I., Zubenko, Y. S., Chernov, A. N., Talabaev, M. V., Demidchik, Y. E., Petkevich, S. K., Kazbanov, V. V., Gurinovich, T. A., Roeva, M. O., Grigoriev, D. G., Kletskov, A. V. \& Kalunov, V. N. Cytotoxic Effects of Chemotherapeutic Drugs and Heterocyclic Compounds at Application on the Cells of Primary Culture of Neuroepithelium Tumors. Med. Chem. 8, 22-32 http://dx.doi.org/10.2174/157340612799278298 (2012)

7. Potkin, V. I., Shcharbin, D., Denisov, A. A., Paschkevich, S. G., Bryszewska, M., Petkevich, S. K., Kletskov, A. V., Lapotko, D. O., Kazbanov, V. V., Gurinovich T. A. \& Kulchitsky V. A. The influence of heterocyclic compound-pamam dendrimer complexes on evoked electrical responses in slices of hypoxic brain tissue. Cell \& Mol. Biol. Lett. 19, 243-248 https://doi.org/10.2478/s11658-014-0193-5 (2014)

8. Kletskov, A. V., Potkin, V. I., Kolesnik, I. A., Petkevich, S. K., Kvachonak, A. V., Dosina, M. O., Loiko, D. O., Larchenko, M. V., Pashkevich, S. G., \& Kulchitsky, V. A. Synthesis and Biological Activity of Novel Comenic Acid Derivatives Containing Isoxazole and Isothiazole Moieties. Nat. Prod. Comm. 13, 1507-1510 https://journals.sagepub.com/doi/pdf/10.1177/1934578X1801301124. (2018)

9. Kulchitsky, V., Zamaro, A., Potkin, V., Suziedelis, K., Koulchitsky, S. \& Kaliadzich, Zh. Perspectives of Conjoint Application of Heterocyclic Compounds and Classical Chemotherapeutic Agents. J. Cancer Sci. Treat, 2, 119-120 https://www.scitcentral.com/article/25/1106/atozJournals.php (2020),

10. Sedletska, Yu., Giraud-Panis, M-J., Malinge, J-M. Cisplatin Is a DNA-Damaging Antitumour Compound Triggering Multifactorial Biochemical Responses in Cancer Cells: Importance of Apoptotic Pathways, Curr. Med. Chem. - Anti-Cancer Agents 5, 251-265 http://dx.doi.org/10.2174/1568011053765967 (2005).

11. Patel, R. V., Park, S. W. An Evolving Role of Piperazine Moieties in Drug Design and Discovery. MiniReviews in Med. Chem. 13, 1579-1601 http://dx.doi.org/10.2174/13895575113139990073 (2013).

12. Kaberdin, R.V., Potkin, V.I., Ol'dekop, Yu.A. The Synthesis and Some Reactions of 3-Trichloromethyl4,5-dichloroisothiazole. Rus. J. Org. Chem. 26, 1347-1352 (1990).

13. Frisch, M. J., Trucks, G. W., Schlegel, H. B., Scuseria, G. E., Robb M.A, Cheeseman J.R, Scalmani G., Barone V., Petersson G.A., Nakatsuji H., Li X., Caricato, M., Marenich, A. V., Bloino J., Janesko B.G., Gomperts R., Mennucci B., Hratchian H.P., Ortiz I.V., Izmaylov, A. F., Sonnenberg, J. L., Williams-Young, D., Ding, F., Lipparini, F., Egidi, F., Goings, J., Peng, B., Petrone, A., Henderson, T., Ranasinghe, D., 
Zakrzewski, V. G., Gao, J., Rega, N., Zheng, G., Liang, W., Hada, M., Ehara, M., Toyota, K., Fukuda, R., Hasegawa, J., Ishida, T., Nakajima, Y., Honda, O., Kitao, H., Nakai, T., Vreven, K., Throssell, J. A., Montgomery, M., Peralta, Jr. J. E., Ogliaro, F., Bearpark, M. J., Heyd, J. J., Brothers, E. N., Kudin, K. N., Staroverov, V. N., Keith, T.,A., Kobayashi, R., Normand, J., Raghavachari, K., Rendell, A. P., Burant, J.,C., lyengar, S.,S., Tomasi, J., Cossi, M., Millam, J. M., Klene, M., Adamo, C., Cammi, R., Ochterski, J. W., Martin, R. I., Morokuma, K., Farkas, O., Foresman, J. B., Fox, D. J. Gaussian 16, Revision B.01. Gaussian, Inc. Wallingford CT, 2016.

14. Becke, A. D. Density-functional thermochemistry. III. The role of exact exchange. J. Chem. Phys. 98, 5648-5652 https://doi.org/10.1063/1.464913. (1993).

15. Lee, Ch., Yang, W., Parr, R. G., Development of the Colle-Salvetti correlation-energy formula into a functional of the electron density. Phys. Rev. B37, 785-789 https://doi.org/10.1103/PhysRevB.37.785 (1988)

16. Tawada, Y., Tsuneda, T., Yanagisawa, S., Yanai, T. \& Hirao, K. A long-range-corrected time-dependent density functional theory. J. Chem. Phys. 120, 8425-8433 https://doi.org/10.1063/1.1688752 (2004).

17. Singh I., El-Emam A. A., Pathak S. K., Srivastava R., Shukla V. K., Prasad O. \& Sinha, L. Experimental and theoretical DFT (B3LYP, X3LYP, CAM-B3LYP and M06-2X) study on electronic structure, spectral features, hydrogen bonding and solvent effects of 4-methylthiadiazole-5-carboxylic acid. Molecular Simulation 45, 1029-1043 https://doi.org/10.1080/08927022.2019.1629434 (2019).

18. Kendall, R. A., Dunning, Jr. T. H., Harrison, R. J. Electron affinities of the first-row atoms revisited. Systematic basis sets and wave functions. J. Chem. Phys. 96, 6796-6806 https://doi.org/10.1063/1.462569 (1992).

19. Dunning, Jr. T. H. Gaussian basis sets for use in correlated molecular calculations. I. The atoms boron through neon and hydrogen. J. Chem. Phys. 90, 1007-1023 https://doi.org/10.1063/1.456153 (1989).

20. Davidson, E. R. Comment on “Comment on Dunning's correlation-consistent basis sets". Chem. Phys. Lett. 260, 514-518 https://doi.org/10.1016/0009-2614(96)00917-7 (1996).

21. Hay, P. J., Wadt, W. R. Ab initio effective core potentials for molecular calculations. Potentials for the transition metal atoms Sc to Hg. J. Chem.. Phys. 82, 270-283 https://doi.org/10.1063/1.448799 (1985).

22. Hay, P. J., Wadt, W. R., Ab initio effective core potentials for molecular calculations. Potentials for $\mathrm{K}$ to Au including the outermost core orbitals. J. Chem. Phys. 82, 299-310 https://doi.org/10.1063/1.448975 (1985).

23. Tomasi, J., Mennucci, B., Cammi, R. Quantum Mechanical Continuum Solvation Models. Chem. Rev. 105, 2999-3094 https://doi.org/10.1021/cr9904009 (2005)

24. Chemcraft - graphical software for visualization of quantum chemistry computations, https://www.chemcraftprog.com. 
25. Tribak, Z., Skalli, M. K., Haoudi, A., Rodi, Y. K. \& Senhaji, O. DFT, Quantum chemical study and biological effects of an heterocyclic molecular. J. Biotech. Biores. 1, JBB.00519. (2019).

26. Dixit, V., Yadav, R. A. Experimental IR and Raman Spectroscopy and DFT Methods Based Material Characterization and Data Analysis of 2-Nitrophenol. Biochem. Pharmacol. (Los Angel) 4, 183 doi:10.4173/2167-0501.1000183. (2015).

27. Kelman, A. D., Peresie, H. J. Mode of DNA binding of cis-platinum(II) antitumor drugs: a base sequence-dependent mechanism is proposed. Cancer Treat. Rep. 63, 1445-1452 https://pubmed.ncbi.nlm.nih.gov/387222. (1979).

28. Siddik, Z. H. Cisplatin: mode of cytotoxic action and molecular basis of resistance. Oncogene 22, 7265-7279 https://doi.org/10.1038/sj.onc.1206933 (2003).

29. Dasari. S., Tchounwou, P. B. Cisplatin in cancer therapy: Molecular mechanisms of action. Eur. J. Pharmacol. 710, 364-378 https://doi.org/10.1016/j.ejphar.2014.07.025 (2014).

30. Murray, J. S., Politzer, P. Molecular electrostatic potentials and noncovalent interactions. WIRES Comput. Mol. Sci. 7, e1326, https://doi.org/10.1002/wcms.1326 (2017).

31. Knaul, F. M., Garcia, P. J., Gospodarowicz, M., Essue, B. M., Lee N. \& Horton R. The Lancet Commission on cancer and health systems: harnessing synergies to achieve solutions. Lancet 398, 1114-1116 https://doi.org/10.1016/s0140-6736(21)01895-x (2021).

\section{Figures}

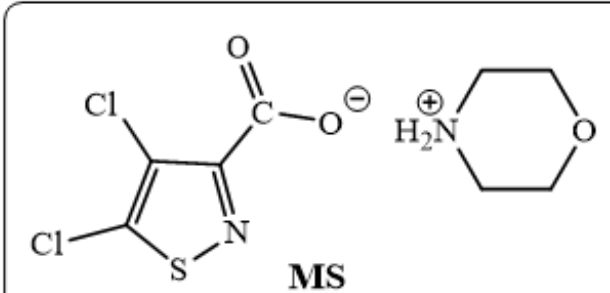

morpholin-4-ium

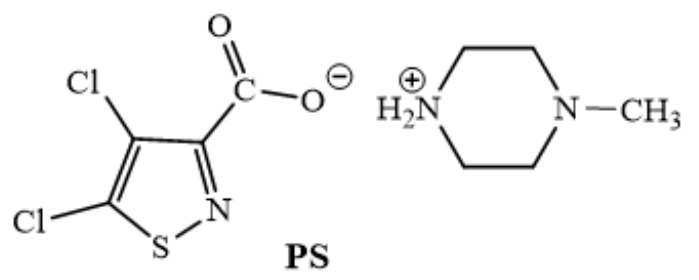

4-methylpiperazin-1-ium

4,5-dichloroisothiazole-3-carboxylate 4,5-dichloroisothiazole-3-carboxylate

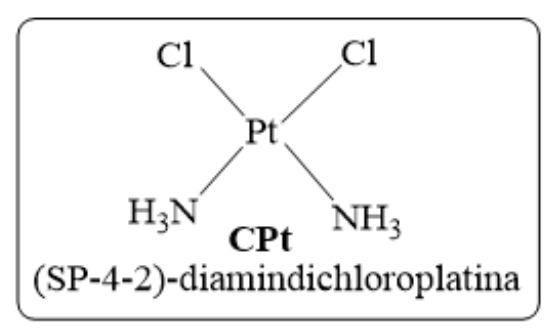

\section{Figure 1}

\section{Structure of substances}<smiles>O=C(O)c1nsc(Cl)c1Cl</smiles>

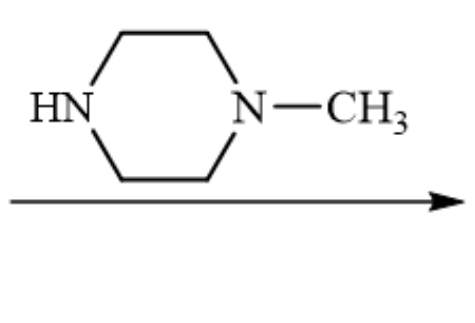<smiles>O=C([O-])c1nsc(Cl)c1Cl</smiles><smiles>CN1CC[NH2+]CC1</smiles> 
Figure 2

Scheme of 4-methylpiperazin-1-ium 4,5-dichloroisothiazole-3-carboxylate (PS) synthesis

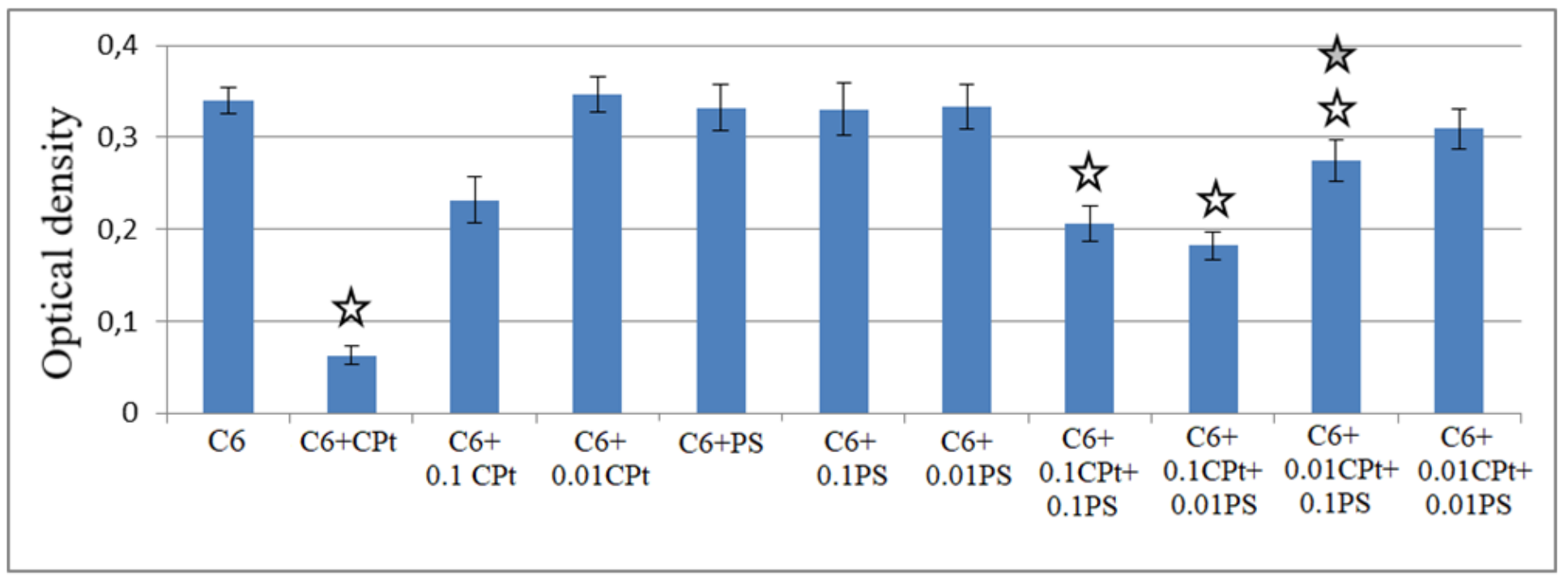

Figure 3

Optical density of $\mathrm{C} 6$ cells culture with addition of cisplatin (CPt) at different concentrations and after subtracting the background value (optical density of DMSO) 

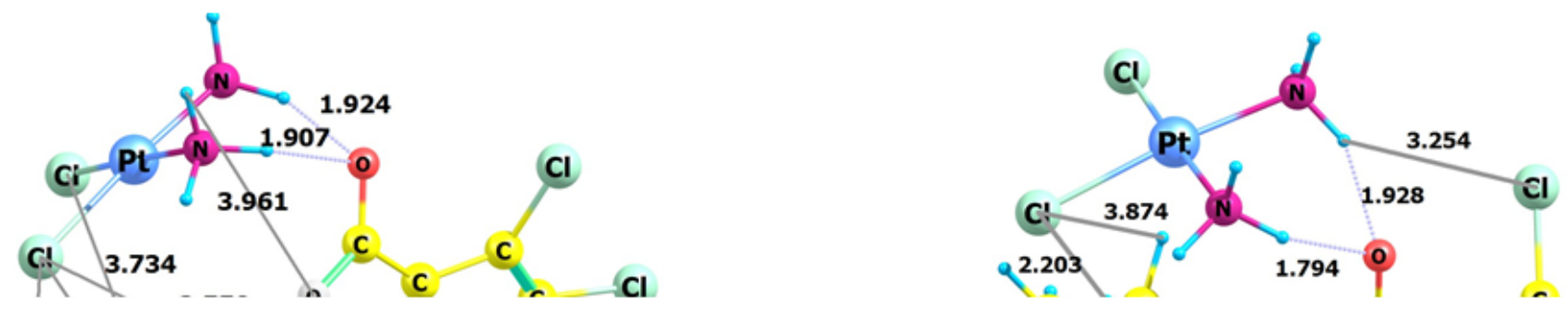

\section{Figure 4}

Calculated optimized structures CPt-MS $(a, b)$ and CPt-PS $(c, d)$ in vacuum $(a, c)$ and with consideration to aqueous medium $(b, d)$ 


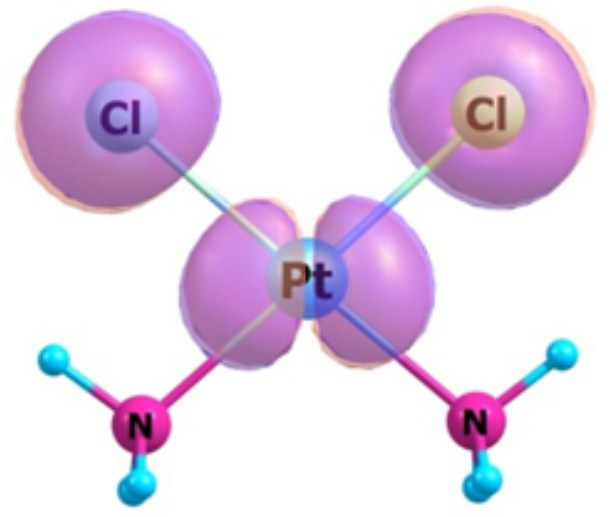

a) $\mathrm{HOMO}$

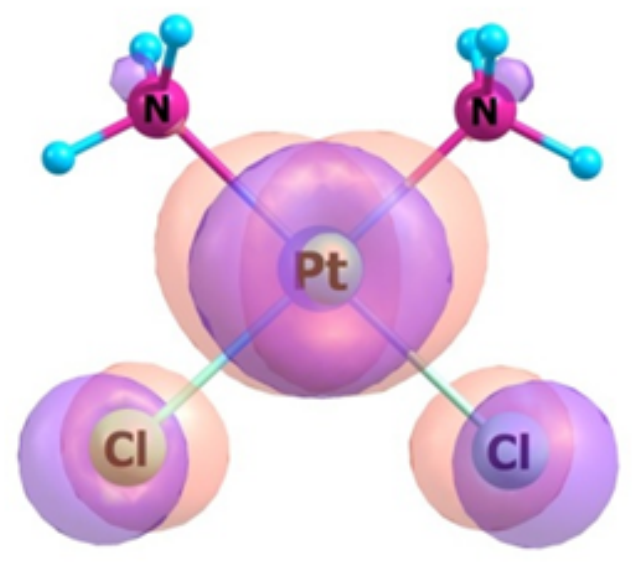

c) $\mathrm{HOMO} \mathrm{W}$

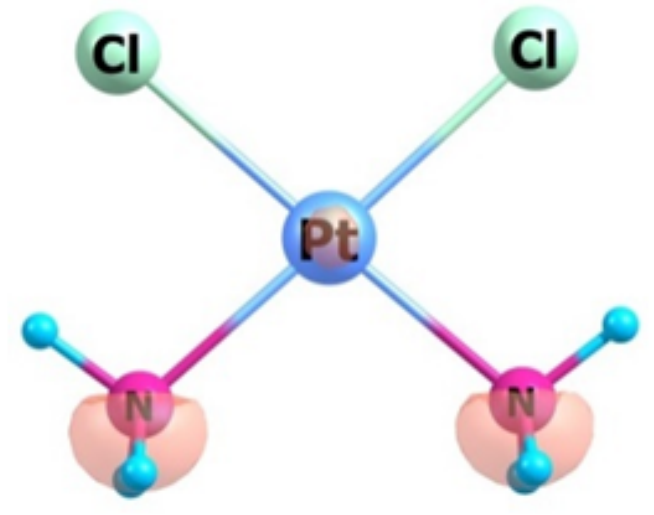

b) LUMO

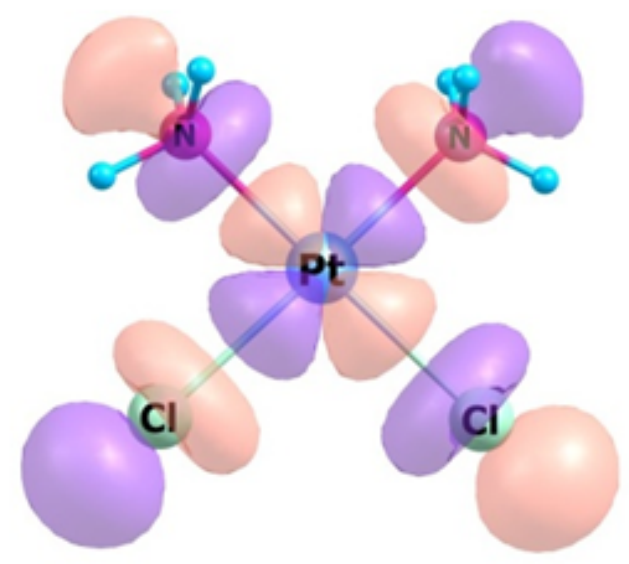

d) LUMO W

Figure 5

Localizations of HOMO and LUMO in cisplatin (CPt) molecule in the form of 3D isosurfaces in vacuum (a, b) and with consideration to water (c, d) 

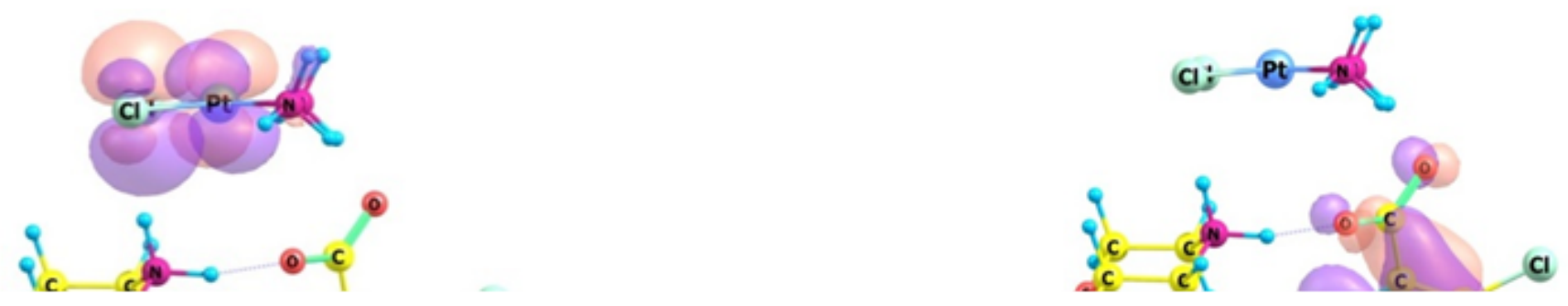

\section{Figure 6}

Localizations of HOMO and LUMO in molecule CPt-MS conjugate in the form of 3D isosurfaces in vacuum $(a, b)$ and with consideration to water $(c, d)$ 

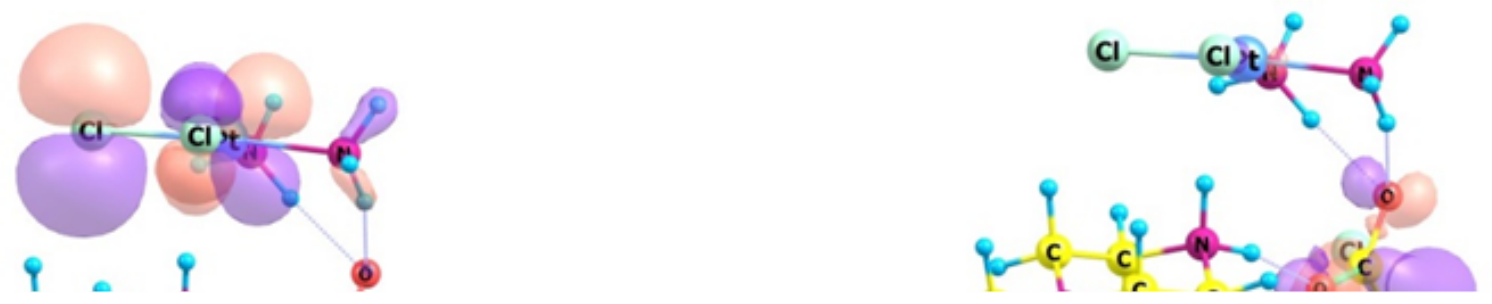

Figure 7

Localizations of HOMO and LUMO in molecule CPt-PS conjugate in the form of $3 \mathrm{D}$ isosurfaces in vacuum $(a, b)$ and with consideration to water $(c, d)$ 

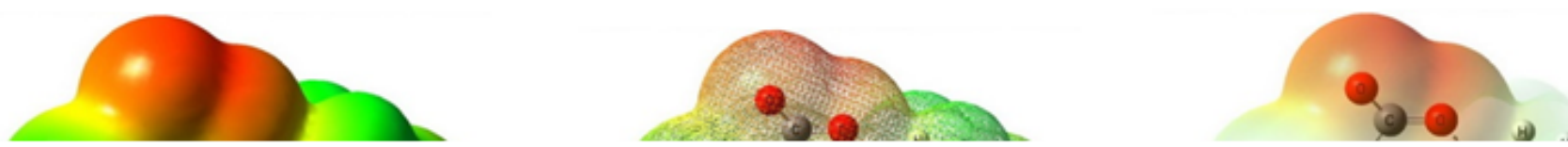

Figure 8

Molecular electrostatic potential diagram of CPt-MS (a-c) CPt-PS (d-f) in different formats 